\title{
An Appliaction of Generalized Fuzzy Soft Matrices in Decision Making Problem
}

\author{
B.K. Saikia ${ }^{1}$, H. Boruah ${ }^{2}$ and P.K. Das ${ }^{3}$ \\ ${ }^{1}$ Principal, L.T.K. College, Azad, North Lakhimpur-787031, Assam \\ ${ }^{2}$ Dept. of Mathematics, Genius Academy, Khelmati, North Lakhimpur-787031, Assam \\ ${ }^{3}$ Dept. of Mathematics, NERIST, Nirjuli-791109, Arunachal Pradesh
}

\begin{abstract}
After development of fuzzy soft matrices, it has been applying in many fields of real life scenarios. The problems which are unable to solve by ordinary matrices can be solved by fuzzy soft matrices. In this paper our main aim is to define generalized fuzzy soft matrices and to study a few of its properties. Finally, we presented a decision making problem based on one of the operation of generalized fuzzy soft matrices.
\end{abstract}

Keywords: Fuzzy Soft Matrices, Generalized Fuzzy Soft Set, Generalized Fuzzy Soft Matrices.

\section{Introduction}

In 1999, Molodtsov [9] introduced the theory of soft sets, which is a new approach to vagueness. In 2003, Maji et al. [11] studied the theory of soft sets initiated by Molodtsov and developed several basic notions of Soft Set Theory. Till now, researchers are contributing a lot on the extension of soft set theory. In 2005, Pei and Miao [6] and Hai Long et al. [6] studied and improved the Maji et al. [11]. Recently Cagman et al. [2, 3] introduced soft matrices and applied it in decision making problems. Researchers published several papers on fuzzy soft matrices and it has been applying in many fields of real life scenarios. B. Chetia and P.K. Das [1] defined Intuitionistic Fuzzy Soft Matrices with different products and properties on these products. In 2010, Majumdar et al. [10 ] generalized the concept of fuzzy soft set introduced by Maji et al. [11] to generalized fuzzy soft set and applied it in decision making problem[8]. Keeping in view, in 2010 Dinda et al. [5] gives the notion of Generalized Intuitionistic Fuzzy Soft Set .They continually work in this field and applied it in decision making problems [4].

In this paper we define generalized fuzzy soft matrix and a few of their operations. Then we study some of its properties. Subsequently based on the operation intersection we have applied generalized fuzzy soft matrices in a decision making problem in medical diagnosis.

\section{Preliminaries}

\section{Definition 2.1 [10]}

Let $U$ be an initial universe, $P(U)$ be the power set of $U, E$ be the set of all parameters and $A \subseteq E$. A pair $\left(f_{A}, E\right)$ is called a soft set over $U$ is defined as the set of ordered pairs $\left(f_{A}, E\right)=\left\{\left(e, f_{A}(e)\right): e \in E, f_{A}(e) \in P(U)\right\}$, where $f_{A}$ is a mapping given by $f_{A}: E \rightarrow P(U)$ such that $f_{A}(e)=\phi$ if $e \notin A$.

Here $f_{A}$ is called approximate function of the soft set $\left(f_{A}, E\right)$. The set $f_{A}(e)$ is called $e$ - approximate value set which consists of related objects of the parameter $e \in E$. In the other words, a soft set over $U$ is a parameterized family of subsets of the universe $U$.

\section{Definition 2.2}

Let $\left(f_{A}, E\right)$ be a fuzzy soft set over $U$, where

$U=\left\{u_{1}, u_{2}, \ldots, u_{m}\right\}$ and $E=\left\{e_{1}, e_{2}, \ldots, e_{n}\right\}, \forall u_{i} \in U, \forall e_{j} \in E$ there exists the membership degree $a_{i j}=f_{e_{j}}\left(u_{i}\right)$ and then we can present all membership degrees by a table as follows: 


\begin{tabular}{l|llll} 
& $e_{1}$ & $e_{2}$ & $\cdots$ & $e_{n}$ \\
\hline$u_{1}$ & $a_{11}$ & $a_{12}$ & $\cdots$ & $a_{1 n}$ \\
$u_{2}$ & $a_{21}$ & $a_{22}$ & $\cdots$ & $a_{2 n}$ \\
$\vdots$ & $\vdots$ & $\vdots$ & $\ddots$ & $\vdots$ \\
$u_{m}$ & $a_{m 1}$ & $a_{m 2}$ & $\cdots$ & $a_{m n}$
\end{tabular}

Then the fuzzy matrix

$$
\tilde{A}_{m \times n}=\left[a_{i j}\right]_{m \times n}=\left(\begin{array}{llll}
a_{11} & a_{12} & \ldots & a_{1 n} \\
a_{21} & a_{22} & \ldots & a_{2 n} \\
\vdots & \vdots & \ddots & \vdots \\
a_{m 1} & a_{m 2} & \ldots & a_{m n}
\end{array}\right)
$$

is called the fuzzy soft matrix of $\left(f_{A}, E\right)$ over $U$.

According to this definition, a fuzzy soft set is uniquely characterized by the fuzzy matrix and vice versa.

\section{Example 2.3}

Let $U=\left\{u_{1}, u_{2}, u_{3}\right\}$ and $A=\left\{e_{1}, e_{2}, e_{3}\right\}$. Then a fuzzy soft set $\left(f_{A}, E\right)$ over $U$ given as below:

$$
\begin{aligned}
& f_{A}\left(e_{1}\right)=\left\{u_{1} / 0.3, u_{2} / 0.5, u_{3} / 0.2\right\} \\
& f_{A}\left(e_{2}\right)=\left\{u_{1} / 0.5, u_{2} / 0.7, u_{3} / 0.6\right\} \\
& f_{A}\left(e_{3}\right)=\left\{u_{1} / 0.4, u_{2} / 0.6, u_{3} / 0.5\right\}
\end{aligned}
$$

Hence the corresponding fuzzy soft matrix $\left[a_{i j}\right]_{m \times n}$ is written by:

$$
\tilde{A}=\left(\begin{array}{llll}
0.3 & 0.5 & 0.4 & 0 \\
0.5 & 0.7 & 0.6 & 0 \\
0.2 & 0.6 & 0.5 & 0
\end{array}\right)
$$

\section{Definition 2.4 [5]}

Let $U=\left\{u_{1}, u_{2}, \ldots, u_{m}\right\}$ be the universal set of elements and $E=\left\{e_{1}, e_{2}, \ldots, e_{n}\right\}$ be the set of parameters. The pair $(U, E)$ will be called soft universe. Let $F: E \rightarrow I^{U}$ and $\lambda$ be a fuzzy subset of $E$ i.e., $\lambda: E \rightarrow I=[0,1], I^{U}$ is the collection of all fuzzy subsets of $U$.

Let $F_{\lambda}: E \rightarrow I^{U} \times I$ be a function define as $F_{\lambda}(e)=(F(e), \lambda(e))$, where $F(e) \in I^{U}$.

Then $F_{\lambda}$ is called the fuzzy soft set over the soft universe $(U, E)$.

Here for each parameters $e_{i}, F_{\lambda}\left(e_{i}\right)=\left(F\left(e_{i}\right), \lambda\left(e_{i}\right)\right)$ indicates not only the degree of belongingness of the elements of $U$ in $F\left(e_{i}\right)$ but also the degree of possibility of such belongingness which is represented by $\lambda\left(e_{i}\right)$.

\section{Example 2.5}

Let $U=\left\{s_{1}, s_{2}, s_{3}, s_{4}\right\}$ be the set of students under consideration and $E=\left\{e_{1}, e_{2}, e_{3}, e_{4}, e_{5}\right\}$ be the set of parameters where $e_{1}=$ expert in 'Mathematics', $e_{2}=$ expert in 'Chemistry, $e_{3}=$ expert in 'Physics', 
$e_{4}=$ expert in 'Biology' and $e_{5}=$ expert in 'English'.

Let $A=\left\{e_{1}, e_{2}, e_{3}, e_{4}\right\} \subseteq E$. Let $\lambda: A \rightarrow I=[0,1]$ be given as:

$\lambda\left(e_{1}\right)=0.8, \lambda\left(e_{2}\right)=0.6, \lambda\left(e_{3}\right)=0.5$ and $\lambda\left(e_{4}\right)=0.7$.

We define $F_{\lambda}$ as follows:

$$
\begin{aligned}
& F_{\lambda}\left(e_{2}\right)=\left(\left\{s_{1} / 0.5, s_{2} / 0.7, s_{3} / 0.4, s_{4} / 0.2\right\}, 0.6\right) \\
& F_{\lambda}\left(e_{3}\right)=\left(\left\{s_{1} / 0.2, s_{2} / 0.3, s_{3} / 0.5, s_{4} / 0.1\right\}, 0.5\right) \\
& F_{\lambda}\left(e_{4}\right)=\left(\left\{s_{1} / 0.3, s_{2} / 0.1, s_{3} / 0.2, s_{4} / 0.4\right\}, 0.7\right)
\end{aligned}
$$

which is the generalized fuzzy soft set representing overall expert of the students.

\section{Generalized Fuzzy Soft Matrix}

Definition 3.1

Let $U$ be an initial universe, $E$ be a set of parameters and $A \subseteq E$. Let $\left(F_{\lambda}, E\right)$ be a generalized fuzzy soft set over $(U, E)$. Then a subset of $U \times E$ is uniquely defined by $R_{A}=\left\{(u, e): e \in A, u \in F_{\lambda}(e)\right\}$ which called a relation form of $\left(F_{\lambda}, E\right)$. The membership function $\mu_{R_{A}}$ and the function $\lambda_{R_{A}}$ are written by $\mu_{R_{A}}: U \times E \rightarrow[0,1]$ and $\lambda_{R_{A}}: U \times E \rightarrow[0,1]$, where $\mu_{R_{A}}:(u, e) \in[0,1]$ is the membership value of $u \in U$ for each $e \in E$ and $\lambda_{R_{A}}:(u, e) \in[0,1]$.

If $\left[\mu_{i j}, \lambda_{j}\right]_{m \times n}=\left(\mu_{R_{A}}\left(u_{i}, e_{j}\right), \lambda_{j}\left(u_{i}, e_{j}\right)\right)$, we define a matrix

$\left[\mu_{i j}, \lambda_{j}\right]_{m \times n}=\left[\begin{array}{cccc}\left(\mu_{11}, \lambda_{1}\right) & \left(\mu_{12}, \lambda_{2}\right) & \cdots & \left(\mu_{1 n}, \lambda_{n}\right) \\ \left(\mu_{21}, \lambda_{1}\right) & \left(\mu_{22}, \lambda_{2}\right) & \cdots & \left(\mu_{2 n}, \lambda_{n}\right) \\ \vdots & \vdots & \vdots & \vdots \\ \left(\mu_{m 1}, \lambda_{1}\right) & \left(\mu_{m 2}, \lambda_{2}\right) & \cdots & \left(\mu_{m n}, \lambda_{n}\right)\end{array}\right]$

which is called an $m \times n$ generalized fuzzy soft matrix (GFSM) of generalized fuzzy soft set(GFSS) $\left(F_{\lambda}, E\right)$. Therefore, we can say that a generalized fuzzy soft set $\left(F_{\lambda}, E\right)$ is uniquely characterized by the matrix $\left[\mu_{i j}, \lambda_{j}\right]_{m \times n}$ and both concepts are interchangeable. The set of all ' $m \times n$, fuzzy soft matrices over $U$ will be denoted by $G F S M_{m \times n}$.

\section{Example 3.2}

Let $U=\left\{u_{1}, u_{2}, u_{3}\right\}$ be the set of the students under consideration for the best academic year with respect the given parameters $E=\left\{e_{1}, e_{2}, e_{3}, e_{4}\right\}$ where $e_{1}=$ result, $e_{2}=$ conduct, $e_{3}=$ attendance and $e_{4}=$ games and sports. Consider $A=\left\{e_{1}, e_{2}, e_{4}\right\} \subseteq E$. Let $\lambda: A \rightarrow[0,1]$ be given as $\lambda\left(e_{1}\right)=0.8, \lambda\left(e_{2}\right)=0.7$ and $\lambda\left(e_{4}\right)=0.6$.

We define $F_{\lambda}$ as follows:

$$
\begin{aligned}
& F_{\lambda}\left(e_{1}\right)=\left(\left\{u_{1} / 0.4, u_{2} / 0.5, u_{4} / 0.6\right\}, 0.8\right), \quad F_{\lambda}\left(e_{2}\right)=\left(\left\{u_{1} / 0.5, u_{2} / 0.7, u_{4} / 0.2\right\}, 0.7\right) \\
& F_{\lambda}\left(e_{3}\right)=\left(\left\{u_{1} / 0.2, u_{2} / 0.3, u_{4} / 0.5\right\}, 0.6\right)
\end{aligned}
$$

We can write the above set as follows: 


\begin{tabular}{l|cccc}
$R_{A}$ & $e_{1}$ & $e_{2}$ & $e_{3}$ & $e_{4}$ \\
$u_{1}$ & $\left(u_{11}, \lambda_{1}\right)$ & $\left(u_{12}, \lambda_{2}\right)$ & $\left(u_{13}, \lambda_{3}\right)$ & $\left(u_{14}, \lambda_{4}\right)$ \\
$u_{2}$ & $\left(u_{21}, \lambda_{1}\right)$ & $\left(u_{22}, \lambda_{2}\right)$ & $\left(u_{23}, \lambda_{3}\right)$ & $\left(u_{24}, \lambda_{4}\right)$ \\
$u_{3}$ & $\left(u_{31}, \lambda_{1}\right)$ & $\left(u_{32}, \lambda_{2}\right)$ & $\left(u_{33}, \lambda_{3}\right)$ & $\left(u_{34}, \lambda_{4}\right)$
\end{tabular}

Then the generalized fuzzy soft matrix can be written as follows:

$$
\left[\mu_{i j}, \lambda_{j}\right]_{3 \times 4}=\left[\begin{array}{cccc}
(0.4,0.8) & (0.5,0.7) & (0,0) & (0.2,0.6) \\
(0.5,0.8) & (0.7,0.7) & (0,0) & (0.3,0.6) \\
(0.6,0.8) & (0.2,0.7) & (0,0) & (0.5,0.6)
\end{array}\right]
$$

\section{Definition 3.3}

$\operatorname{Let}\left[\mu_{i j}, \lambda_{j}\right] \in G F S M_{m \times n}$. Then $\left[\mu_{i j}, \lambda_{j}\right]$ is called

(a) a zero matrix, denoted by [0], if $\mu_{i j}=0, \lambda_{j}=0$ for all $i$ and $j$.

(b) a universal GFSM, denoted by $\left[\mu_{i j}^{\prime}, \lambda_{j}\right]$ if $\mu_{i j}=1, \lambda_{j}=1$ for all $i$ and $j$.

\section{Definition 3.4}

Let $\left[a_{i j}, \lambda_{j}\right],\left[b_{i j}, \lambda_{j}^{\prime}\right] \in \operatorname{GFSM}_{m \times n}$. Then GFSM $\left[c_{i j}, \gamma_{j}\right]$ is called

(a) union of $\left[a_{i j}, \lambda_{j}\right]$ and $\left[b_{i j}, \lambda_{j}^{\prime}\right]$ denoted $\left[a_{i j}, \lambda_{j}\right] \tilde{\cup}\left[b_{i j}, \lambda_{j}^{\prime}\right]$, if $c_{i j}=\min \left\{a_{i j}, b_{i j}\right\}$ and

$$
\gamma_{j}=\min \left\{\lambda_{j}, \lambda_{j}^{\prime}\right\} \text { for all } i \text { and } j \text {. }
$$

(b) intersection of $\left[a_{i j}, \lambda_{j}\right]$ and $\left[b_{i j}, \lambda_{j}^{\prime}\right]$, denoted $\left[a_{i j}, \lambda_{j}\right] \tilde{\cap}\left[b_{i j}, \lambda_{j}^{\prime}\right]$, if $c_{i j}=\min \left\{a_{i j}, b_{i j}\right\}$ and $\gamma_{j}=\max \left\{\lambda_{j}, \lambda_{j}^{\prime}\right\}$ for all $i$ and $j$.

(c) complement of $\left[a_{i j}, \lambda_{j}\right]$, denoted by $\left[a_{i j}, \lambda_{j}\right]^{o}$, if $c_{i j}=1-a_{i j}$ and $\gamma_{j}=1-\lambda_{j}$ for all $i$ and $j$.

\section{Definition 3.5}

Let $\left[a_{i j}, \lambda_{j}\right],\left[b_{i j}, \lambda_{j}^{\prime}\right] \in \operatorname{GFSM}_{m \times n}$.Then $\left[a_{i j}, \lambda_{j}\right]$ and $\left[b_{i j}, \lambda_{j}^{\prime}\right]$ are disjoint, if $\left[a_{i j}, \lambda_{j}\right] \tilde{\cap}\left[b_{i j}, \lambda_{j}^{\prime}\right]=[0]$ for all $i$ and $j$.

\section{Example 3.6}

Let $\left[a_{i j}, \lambda_{j}\right]=\left[\begin{array}{lll}(0.4,0.5) & (0.5,0.7) & (0.2,0.6) \\ (0.5,0.5) & (0.7,0.7) & (0.3,0.6) \\ (0.6,0.5) & (0.2,0.7) & (0.4,0.6)\end{array}\right]$ and $\left[b_{i j}, \lambda_{j}^{\prime}\right]=\left[\begin{array}{lll}(0.7,0.3) & (0.2,0.5) & (0.3,0.4) \\ (0.4,0.3) & (0.3,0.5) & (0.2,0.4) \\ (0.5,0.3) & (0.6,0.5) & (0.5,0.4)\end{array}\right]$

Then $\left[a_{i j}, \lambda_{j}\right] \tilde{\cup}\left[b_{i j}, \lambda_{j}^{\prime}\right]=\left[\begin{array}{lll}(0.7,0.3) & (0.5,0.5) & (0.3,0.4) \\ (0.5,0.3) & (0.7,0.5) & (0.3,0.4) \\ (0.6,0.3) & (0.6,0.5) & (0.5,0.4)\end{array}\right]$

$$
\left[a_{i j}, \lambda_{j}\right] \tilde{\cap}\left[b_{i j}, \lambda_{j}^{\prime}\right]=\left[\begin{array}{ccc}
(0.4,0.5) & (0.2,0.7) & (0.3,0.6) \\
(0.4,0.5) & (0.3,0.7) & (0.2,0.6) \\
(0.5,0.5) & (0.2,0.7) & (0.4,0.6)
\end{array}\right]
$$




$$
\text { and }\left[a_{i j}, \lambda_{j}\right]^{0}=\left[\begin{array}{ccc}
(0.6,0.5) & (0.5,0.3) & (0.8,0.4) \\
(0.5,0.5) & (0.3,0.3) & (0.7,0.4) \\
(0.4,0.5) & (0.8,0.3) & (0.6,0.4)
\end{array}\right]
$$

\section{Proportion 3.7}

Let $\left[a_{i j}, \lambda_{j}\right]$ and $\left[\mathrm{b}_{i j}, \lambda_{j}^{\prime}\right] \in$ GFSM $_{m \times n}$. Then
(a) $\left[\left[a_{i j}, \lambda_{j}\right]^{0}\right]^{0}=\left[a_{i j}, \lambda_{j}\right]$
(b) $\left(\left[a_{i j}, \lambda_{j}\right] \tilde{\cup}\left[\mathrm{b}_{i j}, \lambda_{j}^{\prime}\right]\right)^{0}=\left[a_{i j}, \lambda_{j}\right]^{0} \tilde{\cap}\left[\mathrm{b}_{i j}, \lambda_{j}^{\prime}\right]^{0}$
(c) $\left(\left[a_{i j}, \lambda_{j}\right] \tilde{\cap}\left[\mathrm{b}_{i j}, \lambda_{j}^{\prime}\right]\right)^{0}=\left[a_{i j}, \lambda_{j}\right]^{0} \tilde{\cup}\left[\mathrm{b}_{i j}, \lambda_{j}^{\prime}\right]^{0}$

Proof: (a) $\left[\left[a_{i j}, \lambda_{j}\right]^{0}\right]^{0}=\left[1-a_{i j}, 1-\lambda_{j}\right]^{0}=\left[1-\left(1-a_{i j}\right), 1-\left(1-\lambda_{j}\right)\right]=\left[a_{i j}, \lambda_{j}\right]$

(b) $\left(\left[a_{i j}, \lambda_{j}\right] \tilde{\cap}\left[b_{i j}, \lambda_{j}^{\prime}\right]\right)^{0}=\left[\min \left\{a_{i j}, b_{i j}\right\}, \max \left\{\lambda_{j}, \lambda_{j}^{\prime}\right\}\right]^{0}$

$$
\begin{aligned}
& =\left[1-\min \left\{a_{i j}, b_{i j}\right\}, 1-\max \left\{\lambda_{j}, \lambda_{j}^{\prime}\right\}\right] \\
= & {\left[\max \left\{1-a_{i j}, 1-b_{i j}\right\}, \min \left\{1-\lambda_{j}, 1-\lambda_{j}^{\prime}\right\}\right] } \\
& =\left[1-a_{i j}, 1-\lambda_{j}\right] \tilde{\cup}\left[1-b_{i j}, 1-\lambda_{j}^{\prime}\right] \\
= & {\left[a_{i j}, \lambda_{j}\right]^{0} \tilde{\cup}\left[b_{i j}, \lambda_{j}^{\prime}\right]^{0} }
\end{aligned}
$$

(c) $\left(\left[a_{i j}, \lambda_{j}\right] \widetilde{\cup}\left[b_{i j}, \lambda_{j}^{\prime}\right]\right)^{0}=\left[\max \left\{a_{i j}, b_{i j}\right\}, \min \left\{\lambda_{j}, \lambda_{j}^{\prime}\right\}\right]^{0}$

$$
\begin{aligned}
& =\left[1-\max \left\{a_{i j}, b_{i j}\right\}, 1-\min \left\{\lambda_{j}, \lambda_{j}^{\prime}\right\}\right] \\
& =\left[\min \left\{1-a_{i j}, 1-b_{i j}\right\}, \max \left\{1-\lambda_{j}, 1-\lambda_{j}^{\prime}\right\}\right] \\
& =\left[1-a_{i j}, 1-\lambda_{j}\right] \tilde{\cap}\left[1-b_{i j}, 1-\lambda_{j}^{\prime}\right] \\
& =\left[a_{i j}, \lambda_{j}\right]^{0} \tilde{\cap}\left[b_{i j}, \lambda_{j}^{\prime}\right]^{0}
\end{aligned}
$$

\section{Proportion 3.8}

Let $\left[a_{i j}, \lambda_{j}\right],\left[b_{i j}, \gamma_{j}\right]$ and $\left[c_{i j}, \varphi_{j}\right] \in \operatorname{GFSM}_{m \times n}$. Then

(i) $\left[a_{i j}, \lambda_{j}\right] \tilde{\cap}\left[b_{i j}, \gamma_{j}\right]=\left[b_{i j}, \lambda_{j}\right] \tilde{\cap}\left[a_{i j}, \gamma_{j}\right]$

(ii) $\left[a_{i j}, \lambda_{j}\right] \tilde{\cup}\left[b_{i j}, \gamma_{j}\right]=\left[b_{i j}, \lambda_{j}\right] \tilde{\cup}\left[a_{i j}, \gamma_{j}\right]$

(iii) $\left[a_{i j}, \lambda_{j}\right] \tilde{\cap}\left(\left[b_{i j}, \gamma_{j}\right] \tilde{\cap}\left[c_{i j}, \varphi_{j}\right]\right)=\left(\left[a_{i j}, \lambda_{j}\right] \tilde{\cap}\left[b_{i j}, \gamma_{j}\right]\right) \tilde{\cap}\left[c_{i j}, \varphi_{j}\right]$

(iv) $\left[a_{i j}, \lambda_{j}\right] \tilde{\cup}\left(\left[b_{i j}, \gamma_{j}\right] \tilde{\cup}\left[c_{i j}, \varphi_{j}\right]\right)=\left(\left[a_{i j}, \lambda_{j}\right] \tilde{\cup}\left[b_{i j}, \gamma_{j}\right]\right) \tilde{\cup}\left[c_{i j}, \varphi_{j}\right]$

(v) $\left[a_{i j}, \lambda_{j}\right] \tilde{\cup}\left(\left[b_{i j}, \gamma_{j}\right] \tilde{\cap}\left[c_{i j}, \varphi_{j}\right]\right)=\left(\left[a_{i j}, \lambda_{j}\right] \tilde{\cup}\left[b_{i j}, \gamma_{j}\right]\right) \tilde{\cap}\left(\left[a_{i j}, \lambda_{j}\right] \tilde{\cup}\left[c_{i j}, \varphi_{j}\right]\right)$

(vi) $\left[a_{i j}, \lambda_{j}\right] \tilde{\cap}\left(\left[b_{i j}, \gamma_{j}\right] \tilde{\cup}\left[c_{i j}, \varphi_{j}\right]\right)=\left(\left[a_{i j}, \lambda_{j}\right] \tilde{\cap}\left[b_{i j}, \gamma_{j}\right]\right) \tilde{\cup}\left(\left[a_{i j}, \lambda_{j}\right] \tilde{\cap}\left[c_{i j}, \varphi_{j}\right]\right)$

\section{Product of Generalized Fuzzy Soft Matrices}

In these section we define four different product of Generalized Intuitionistic Fuzzy Soft Matrices. Cagman et al. [2] defined four different types of product of fozzy soft matrices. We extend these four products to Generalized Intuitionistic Fuzzy Soft Matrices and study some of its properties.

\section{Definition 4.1}

Let $\left[a_{i j}, \lambda_{j}\right]$ and $\left[b_{i k}, \gamma_{k}\right] \in \operatorname{GFSM}_{m \times n}$. Then And-product of $\left[a_{i j}, \lambda_{j}\right]$ and $\left[b_{i k}, \gamma_{k}\right]$ is defined by $\wedge: G F S M_{m \times n} \times$ GFSM $_{m \times n} \rightarrow \operatorname{GFSM}_{m \times n^{2}}$ and $\left[a_{i j}, \lambda_{j}\right] \wedge\left[b_{i k}, \gamma_{k}\right]=\left[c_{i p}, \psi_{p}\right]$ 
where $c_{i p}=\min \left\{a_{i j}, b_{i k}\right\}$ and $\psi_{p}=\max \left\{\lambda_{j}, \gamma_{k}\right\}$ such that $p=n(j-1)+k$.

\section{Definition 4.2}

Let $\left[a_{i j}, \lambda_{j}\right]$ and $\left[b_{i k}, \gamma_{k}\right] \in G F S M_{m \times n}$. Then Or-product of $\left[a_{i j}, \lambda_{j}\right]$ and $\left[b_{i k}, \gamma_{k}\right]$ is defined by

$$
\vee: G F S M_{m \times n} \times G_{F S M_{m \times n}} \rightarrow \operatorname{GFSM}_{m \times n^{2}} \text { and }\left[a_{i j}, \lambda_{j}\right] \vee\left[b_{i k}, \gamma_{k}\right]=\left[c_{i p}, \psi_{p}\right]
$$

where $c_{i p}=\max \left\{a_{i j}, b_{i k}\right\}$ and $\psi_{p}=\min \left\{\lambda_{j}, \gamma_{k}\right\}$ such that $p=n(j-1)+k$.

\section{Definition 4.3}

Let $\left[a_{i j}, \lambda_{j}\right]$ and $\left[b_{i k}, \gamma_{k}\right] \in G_{F S M_{m \times n}}$.Then And-Not-product of $\left[a_{i j}, \lambda_{j}\right]$ and $\left[b_{i k}, \gamma_{k}\right]$ is defined by

$$
\bar{\wedge}: G F S M_{m \times n} \times G F S M_{m \times n} \rightarrow \operatorname{GFSM}_{m \times n^{2}} \text { and }\left[a_{i j}, \lambda_{j}\right] \bar{\wedge}\left[b_{i k}, \gamma_{k}\right]=\left[c_{i p}, \psi_{p}\right]
$$

where $c_{i p}=\min \left\{a_{i j}, 1-b_{i k}\right\}$ and $\psi_{p}=\max \left\{\lambda_{j}, 1-\gamma_{k}\right\}$ such that $p=n(j-1)+k$.

\section{Definition 4.4}

Let $\left[a_{i j}, \lambda_{j}\right]$ and $\left[b_{i k}, \gamma_{k}\right] \in \operatorname{GFSM}_{m \times n}$. Then Or-Not-product of $\left[a_{i j}, \lambda_{j}\right]$ and $\left[b_{i k}, \gamma_{k}\right]$ is defined by

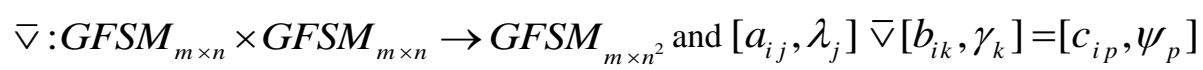

where $c_{i p}=\max \left\{a_{i j}, 1-b_{i k}\right\}$ and $\psi_{p}=\min \left\{\lambda_{j}, 1-\gamma_{k}\right\}$ such that $p=n(j-1)+k$.

\section{Example 4.5}

Assume that $\left[a_{i j}, \lambda_{j}\right],\left[b_{i k}, \gamma_{k}\right] \in \operatorname{GFSM}_{2 \times 3}$ are given as follows:

$$
\left[a_{i j}, \lambda_{j}\right]=\left[\begin{array}{ccc}
(0.4,0.5) & (0.5,0.7) & (0.2,0.6) \\
(0.5,0.5) & (0.7,0.7) & (0.3,0.6) \\
(0.6,0.5) & (0.2,0.7) & (0.4,0.6)
\end{array}\right] \text { and }\left[b_{i k}, \gamma_{k}\right]=\left[\begin{array}{ccc}
(0.7,0.3) & (0.2,0.5) & (0.3,0.4) \\
(0.4,0.3) & (0.3,0.5) & (0.2,0.4) \\
(0.5,0.3) & (0.6,0.5) & (0.5,0.4)
\end{array}\right]
$$

Then

$$
\begin{aligned}
& {\left[a_{i j}, \lambda_{j}\right] \wedge\left[b_{i j}, \gamma_{k}\right]} \\
& =\left[\begin{array}{llllllllll}
(0.4,0.5) & (0.2,0.5) & (0.3,0.5) & (0.5,0.7) & (0.2,0.7) & (0.2,0.7) & (0.2,0.6) & (0.2,0.6) & (0.2,0.6) \\
(0.4,0.5) & (0.3,0.5) & (0.2,0.5) & (0.4,0.7) & (0.3,0.7) & (0.2,0.7) & (0.3,0.6) & (0.3,0.6) & (0.2,0.6) \\
(0.5,0.5) & (0.6,0.5) & (0.5,0.5) & (0.2,0.7) & (0.2,0.7) & (0.2,0.7) & (0.4,0.6) & (0.4,0.6) & (0.4,0.6)
\end{array}\right]
\end{aligned}
$$

Similarly, we can alsofind the other products $\left[a_{i j}, \lambda_{j}\right] \vee\left[b_{i j}, \gamma_{k}\right],\left[a_{i j}, \lambda_{j}\right] \nabla\left[b_{i j}, \gamma_{k}\right]$ and $\left[a_{i j}, \lambda_{j}\right] \wedge\left[b_{i j}, \gamma_{k}\right]$ of generalized fuzzy soft matrices.

Note that commutativity is not valid for the product of generalized fuzzy soft matrices.

\section{Proposition 4.6}

Let $\left[a_{i j}, \lambda_{j}\right]$ and $\left[b_{i k}, \gamma_{k}\right] \in$ GFSM $_{m \times n}$. Then the following are true

$$
\begin{aligned}
& \text { (i) }\left(\left[a_{i j}, \lambda_{j}\right] \vee\left[b_{i j}, \gamma_{k}\right]\right)^{0}=\left[a_{i j}, \lambda_{j}\right]^{0} \wedge\left[b_{i j}, \gamma_{k}\right]^{0} \\
& \text { (ii) }\left(\left[a_{i j}, \lambda_{j}\right] \wedge\left[b_{i j}, \gamma_{k}\right]\right)^{0}=\left[a_{i j}, \lambda_{j}\right]^{0} \vee\left[b_{i j}, \gamma_{k}\right]^{0} \\
& \text { (iii) }\left(\left[a_{i j}, \lambda_{j}\right] \nabla\left[b_{i j}, \gamma_{k}\right]\right)^{0}=\left[a_{i j}, \lambda_{j}\right]^{0} \pi\left[b_{i j}, \gamma_{k}\right]^{0} \\
& \text { (iv) }\left(\left[a_{i j}, \lambda_{j}\right] \bar{\wedge}\left[b_{i j}, \gamma_{k}\right]\right)^{0}=\left[a_{i j}, \lambda_{j}\right]^{0} \nabla\left[b_{i j}, \gamma_{k}\right]^{0}
\end{aligned}
$$




\section{A Generalized Fuzzy Matrices Based Decision Making Problem}

Let more than one decision makers want to take a decision or to select an object jointly from the ' $\mathrm{m}$ ' number of objects which have ' $n$ ' number of features i.e., parameters $(E)$. Suppose that each decision maker has freedom to take his decision of inclusion and evaluation of parameters associated with the selected object or may choice the same set of parameters i.e., each decision maker has his own choice parameters belonging to the parameter set $E$ and has his own view of evaluation. Here it is assumed that the parameter evaluation of the objects by the decision makers must be generalized fuzzy and may be presented in linguistic form or generalized fuzzy soft set format, alternatively, in the form of generalized fuzzy soft matrices. Now the aim of the decision makers is to find out the object out of ' $m$ ' object jointly as far as possible.

\section{Definition 5.1}

Let $\left[c_{i j}, \gamma_{j}\right] \in \tilde{A} \tilde{\cap} \tilde{B}$ where $\tilde{A}$ and $\tilde{B}$ are two generalized fuzzy soft matrices. Then the set $W\left(u_{i}\right)=\left\{u_{i} \in U \mid \sum c_{i j}\right\}$ is called weight for each $u_{i} \in U$.

\section{Procedure of solving problem:}

A real life problem can be solved by using different Mathematical methods. The decision maker can choose the easiest method from the alternative. After taking decision by the decision makers that, they solve a particular problem by using the operation of generalized soft matrices, the decision makers go through the following algorithm.

\section{Algorithm:}

Step 1: To construct the generalized fuzzy soft matrices with respect to their own choice parameters of the decision makers.

Step 2: To compute the union or intersection of generalised fuzzy soft matrices.

Step 3: To compute the weight of each object $\left(O_{i}\right)$ by adding the membership values of the entries of its concerned row ( $i^{\text {th }}$-row) of the union or intersection of generalised fuzzy soft matrices.

Step 4: The object having the highest weight becomes the optimal choice object.

To illustrate the basic idea of the GFSM-algorithm, now we apply it to the following generalized fuzzy soft set (or generalized fuzzy soft matrix) based decision making problems.

\section{Case Study:}

Generally in medical science a patient suffering from a disease may have multiple symptoms. Again it is also observed that there are certain symptoms which may be common to more than one diseases leading to diagnostic dilemma. Sometimes the doctor has to face many problems when an area is largely affected new disease. Then the doctor has detected the disease commencing the common symptoms of the patients.

Let $U=\left\{p_{1}, p_{2}, p_{3}, p_{4}\right\}$ be the set of patients and $E=\left\{e_{1}, e_{2}, e_{3}, e_{4}, e_{5}, e_{6}, e_{7}, e_{8}\right\}$ be the set of parameters of symptoms of dengue where

$$
\begin{aligned}
& e_{1}=\text { high fever }\left(104 \mathrm{~F}, 40^{\circ} \mathrm{C}\right), e_{2}=\text { headache, } e_{3}=\text { extreme fatigue } \\
& e_{4}=\text { red eyes and pain in the eyes, } e_{5}=\text { enlarged lymph nodes, } \\
& e_{6}=\text { deep muscle and joint pains (during first hours of illness) } \\
& e_{7}=\text { nausea and vomiting, } e_{8}=\text { low pressure and heart rate. }
\end{aligned}
$$

Suppose two Dr. X and Dr. Y examines the patients on based on the same set of parameters.

Let $\lambda: E \rightarrow[0,1]$. They consider the function on the parameters as follows:

Dr. $\mathrm{X}$ grade on the parameters as 
$\lambda\left(e_{1}\right)=0.7, \lambda\left(e_{2}\right)=0.4, \lambda\left(e_{3}\right)=0.3, \lambda\left(e_{4}\right)=0.5, \lambda\left(e_{5}\right)=0.6, \lambda\left(e_{6}\right)=0.5, \lambda\left(e_{7}\right)=0.7, \lambda\left(e_{8}\right)=0.2$ and the Dr. Y grade on the parameters as

$\lambda\left(e_{1}\right)=0.7, \lambda\left(e_{2}\right)=0.5, \lambda\left(e_{3}\right)=0.4, \lambda\left(e_{4}\right)=0.3, \lambda\left(e_{5}\right)=0.6, \lambda\left(e_{6}\right)=0.4, \lambda\left(e_{7}\right)=0.7, \lambda\left(e_{8}\right)=0.3$.

Step 1: Based on these functions the two doctors construct generalized matrices for the patients $\tilde{A}$ and $\tilde{B}$ respectively given as follows:

$$
\begin{aligned}
& \tilde{A}=\begin{array}{l}
p_{1} \\
p_{2} \\
p_{3} \\
p_{4}
\end{array}\left[\begin{array}{lllllllll}
(0.7,0.8) & (0.6,0.6) & (0.8,0.3) & (0.6,0.5) & (0.7,0.4) & (0.5,0.5) & (0.7,0.7) & (0.4,0.2) \\
(0.6,0.8) & (0.5,0.6) & (0.3,0.3) & (0.1,0.5) & (0.1,0.4) & (0.6,0.5) & (0.2,0.7) & (0.5,0.2) \\
(0.3,0.8) & (0.7,0.6) & (0.5,0.3) & (0.3,0.5) & (0.4,0.4) & (0.2,0.5) & (0.3,0.7) & (0.5,0.2)
\end{array}\right] \\
& \tilde{B}=\begin{array}{l}
p_{1} \\
p_{2} \\
p_{3} \\
p_{4}
\end{array}\left[\begin{array}{lllllllll}
(0.7,0.7) & (0.6,0.5) & (0.8,0.4) & (0.6,0.3) & (0.7,0.6) & (0.6,0.4) & (0.8,0.7) & (0.5,0.3) \\
(0.5,0.7) & (0.2,0.5) & (0.4,0.4) & (0.4,0.3) & (0.2,0.6) & (0.5,0.4) & (0.3,0.7) & (0.4,0.3) \\
(0.2,0.7) & (0.4,0.5) & (0.3,0.4) & (0.2,0.3) & (0.2,0.6) & (0.1,0.4) & (0.4,0.7) & (0.3,0.3)
\end{array}\right] \\
& \tilde{A} \tilde{\cap} \tilde{B}=\left[\begin{array}{llllllll}
(0.7,0.8) & (0.6,0.6) & (0.8,0.4) & (0.6,0.5) & (0.7,0.6) & (0.5,0.5) & (0.7,0.7) & (0.4,0.3) \\
(0.5,0.8) & (0.2,0.6) & (0.3,0.4) & (0.1,0.5) & (0.1,0.6) & (0.5,0.5) & (0.2,0.7) & (0.4,0.3) \\
(0.4,0.8) & (0.3,0.6) & (0.2,0.4) & (0.1,0.5) & (0.2,0.6) & (0.2,0.5) & (0.2,0.7) & (0.2,0.3) \\
(0.2,0.8) & (0.4,0.6) & (0.3,0.4) & (0.2,0.5) & (0.2,0.6) & (0.1,0.5) & (0.3,0.7) & (0.3,0.3)
\end{array}\right]
\end{aligned}
$$

Step 3: Now the weights of the patients are respectively,

$$
\begin{aligned}
& W\left(p_{1}\right)=0.7+0.6+0.8+0.6+0.7+0.5+0.7+0.4=5.0 \\
& W\left(p_{2}\right)=0.5+0.2+0.3+0.1+0.1+0.5+0.2+0.3=2.2 \\
& W\left(p_{3}\right)=0.4+0.3+0.2+0.1+0.2+0.2+0.2+0.2=1.8 \\
& W\left(p_{4}\right)=0.2+0.4+0.3+0.2+0.2+0.1+0.3+0.3=2.0
\end{aligned}
$$

Step 4: The maximum weight (5.0) obtains by patient is $p_{1}$.

Hence the patient $p_{1}$ has suffered in dengue.

\section{Conclusion}

In this paper, we have introduced generalized fuzzy soft matrices and some of their properties. We also defined product of generalized fuzzy soft matrices and some of their properties. Then we defined weighted generalized fuzzy soft fuzzy soft sets. Finally we presented an application of generalized soft matrices in medical diagnosis.

\section{References}

[1] Chetia, B. and Das, P. K. : "Some Results of Intuitionistic Fuzzy Soft matrix Theory," Pelagia Research Library, Advances in Applied Science Research, 2012,Vol. 3(1)412- 423.

[2] Cagman, N. and Enginoglu, S. :"Fuzzy soft Matrix Theory and Its Application in Decision Making," Iranian Journal of Fuzzy systems, Vol.9(1), 2012.

[3] Cagman, N., Enginoglu, S. : "Soft Matrix Theory and It's Decision Making," Computers and Mathematics with applications, 59(2010), 3308-3314.

[4] Dinda, B., Bera, T. and Samanta, T. K. : “Generalized Intuitionistic Fuzzy Soft Sets and An Adjustable Approach to Decision Making," Annals of Fuzzy Mathematics and Informatics, Vol. 4, No. 2, 2012, pp. 207-215.

[5] Dinda, B., Bera, T. and Samanta, T. K. : “Generalized Intuitionistic Fuzzy Soft Sets and Its Application in Decision Making," [math.GM], 2010.

[6] D. Pei and D. Miao: "From Soft Sets to Information Systems," Proceedings of the IEEE International Conference on Granular Computing 2 (2005), 617-621. 
[7] Hai Long YANG: "Notes on Generalised Fuzzy Soft Sets," Journal of Mathematical Research \& Exposition, 2011, Vol. 31, No. 3, pp. 567-570.

[8] Majumdar, P., Samanta, T. K.: “A Generalized Fuzzy Soft Set Based Student Ranking System,” International Journal Advance Soft Computer Application, Vol. 3, 2011.

[9] Molodtsov, D.: "Soft Set Theory - First Result," Computers and Mathematics with Applications 37 (1999), $19-31$.

[10] P. Majumdar, S. K. Samanta : "Generalized Fuzzy Soft Sets," Computers and Mathematics with Applications, 59(2010), pp.14251432.

[11] P. K. Maji and A. R. Roy: "Soft Set Theory," Computers and Mathematics with Applications, 45 (2003), $555-562$.

\section{Acknowledgments:}

The work is supported by the University Grants Commission (UGC) as Major Research Project No. F. 41-772/2012(SR) Dt. 17 July, 2012. 